\title{
Role of the ipsilateral motor cortex in mirror movements
}

\author{
Tadashi Kanouchi, Takanori Yokota, Fumiko Isa, Kenji Ishii, Michio Senda
}

Department of

Neurology, Tokyo

Medical and Dental

University

T Kanouchi

T Yokota

Department of

Neurology, Tokyo

Metropolitan Kita

Medical Rehabilitation

Center

F Isa

Positron Medical

Center, Tokyo

Metropolitan Institute

of Gerontology, Tokyo,

Japan 1-5-45,

Yushima, Bunkyo-ku,

Tokyo 113, Japan

K Ishii

$M$ Senda

Correspondence to:

Dr Takanori Yokota,

Department of Neurology,

Tokyo Medical and Dental

University, 1-5-45, Yushima,

Bunkyo-ku, Tokyo 113,

Japan.

Received 1 August 1996

and in revised form

2 January 1997

Accepted 23 January 1997

\begin{abstract}
The mechanism of mirror movements in two patients was investigated; one with congenital mirror movement, the other with schizencephaly. Transcranial magnetic stimulation on one side elicited motor evoked potentials (MEPs) in their thenar muscles on both sides with almost the same latencies, minimal thresholds, and cortical topographies. During voluntary contraction of the thenar muscle on one side, contralateral transcranial magnetic stimulation induced a silent period not only on the voluntary contraction side but on the mirror movement side and of the same duration. By contrast, ipsilateral transcranial magnetic stimulation elicited MEPs without silent periods in both muscles. With intended unilateral finger movements, an $\mathrm{H}_{2}{ }^{15} \mathrm{O}-\mathrm{PET}$ activation study showed that the regional cerebral blood flow increased predominantly in the contralateral sensorimotor cortex, as seen in normal subjects, although mirror movements occurred.

It is considered that the ipsilateral motor cortex plays a major part in the generation of mirror movements, which may be induced through the ipsilateral uncrossed corticospinal tract.
\end{abstract}

(F Neurol Neurosurg Psychiatry 1997;62:629-632)

Keywords: mirror movement; transcranial magnetic stimulation; silent period; $\mathrm{H}_{2}{ }^{15} \mathrm{O}-\mathrm{PET}$

Mirror movements are symmetric, identical, contralateral involuntary movements that accompany voluntary movements on one side. They are common in young children and usually disappear around the age of 10 years. Persistent mirror movements in adults have been seen with Kallmann's syndrome, ${ }^{12}$ Klippel-Feil syndrome, ${ }^{3}$ and congenital hemiparesis, ${ }^{4}$ as well as in persons without any other neurological abnormality (congenital mirror movement). ${ }^{56}$ In some of these cases, bilateral motor cortical activities during intended unilateral movements have been shown in reports of movement related cortical potentials ${ }^{2}$ and in a PET activation study. ${ }^{6}$

We investigated the mirror movements of two patients by transcranial magnetic stimulation and PET techniques.

\section{Case reports}

Patient 1 was a 16 year old, ambidextrous boy with congenital mirror movement. He had no neurological abnormalities except these movements. Brain MRI was normal. His family history was negative for mirror movements. Patient 2 was a 37 year old, left handed man with schizencephaly. He had tetraparesis, predominantly on the right side. Brain MRI showed clefts in both hemispheres; one in the right central region and a more severe one in the left frontal region, which communicated with the lateral ventricles. In both patients slight to moderate mirror movements occurred only in the two upper limbs, predominantly in the distal parts.

\section{Methods}

We studied the mirror movements in the left hand of patient 1 , which appeared during voluntary right hand movement, and those in the right hand of patient 2. All EMG data were recorded from surface electrodes placed on both thenar muscles using a bandpass filter of 10 to $3000 \mathrm{~Hz}$.

TRANSCRANIAL MAGNETIC STIMULATION Transcranial magnetic stimulation was carried out with a Magstim 200 (the Magstim Company, Witland, Dyfed, UK) through a figure of eight coil with a $9 \mathrm{~cm}$ outer diameter in each loop of the figure of eight and which produces a peak magnetic field of $2.2 \mathrm{~T}$; transcranial magnetic stimulation at $80 \%$ to $100 \%$ intensity of the maximum stimulator output was given over the left hand motor area with the coil handle pointing backward for patient 1 and over the right hand motor area with the coil handle pointing posterolaterally for patient 2. We estimated the latency of the rectified and averaged motor evoked potentials (MEPs) in each relaxed muscle and the minimal threshold intensity for eliciting MEP.

Topographical maps of the cortical representation of the MEPs in the relaxed thenar muscles in both patients were made from the MEP sizes evoked by transcranial magnetic stimulation at $120 \%$ the intensity of the mini- 


\section{The topographical maps for MEPs}
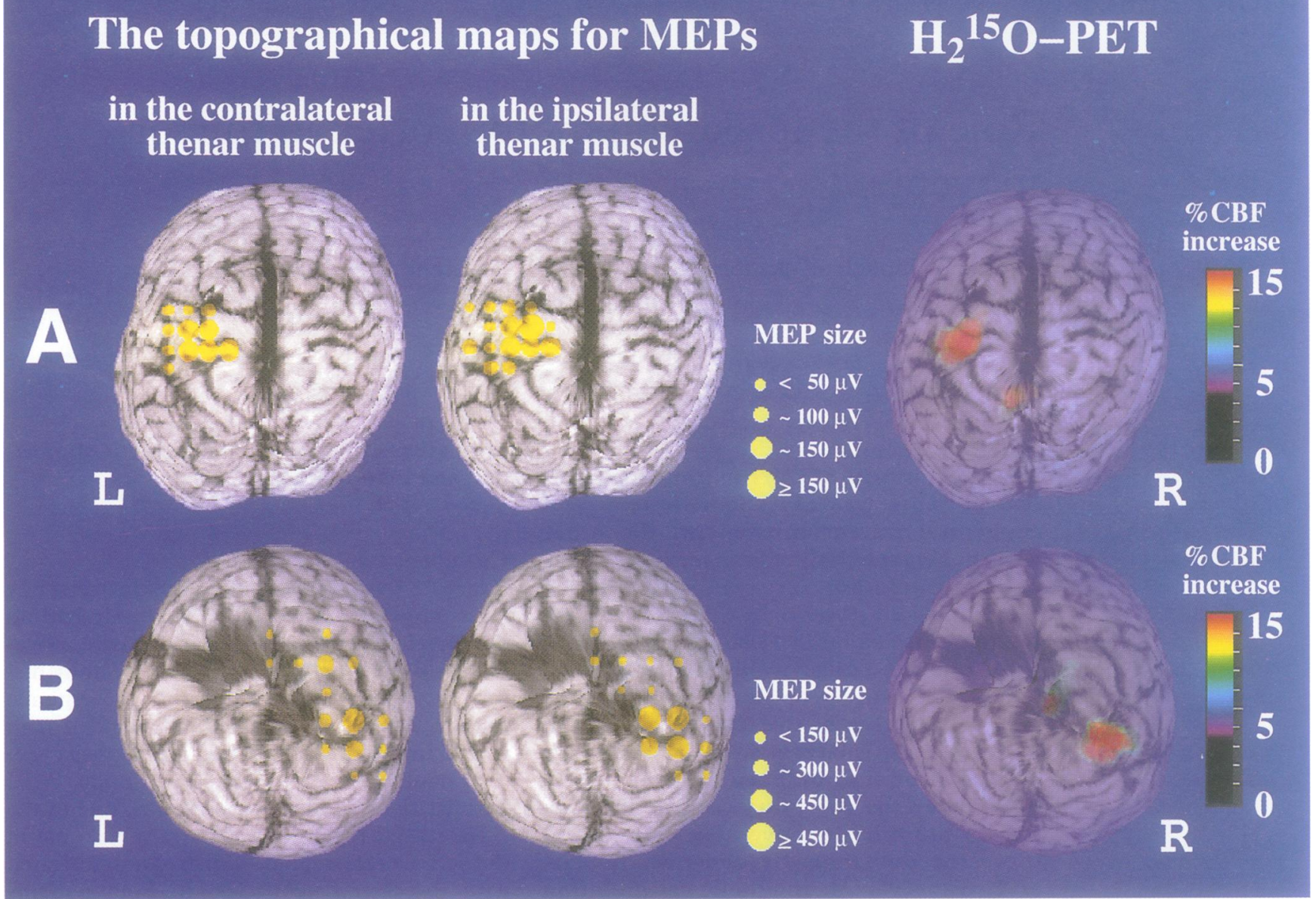

Figure 1 Topographical cortical maps for MEPs in the thenar muscles of both patients (left: thenar muscle contralateral to transcranial magnetic stimulation; middle: thenar muscle ipsilateral to transcranial magnetic stimulation and right: subtracted $\mathrm{H}_{2}{ }^{15} \mathrm{O}-\mathrm{PET}$ images between the unilateral motor task and resting control conditions. All are superimposed on the patients'own MRIs (A: patient 1, B: patient 2). Yellow circles in the topographical maps are the sites at which transcranial magnetic stimulation elicited MEPs. Circle size shows the mean MEP amplitude. In both patients, an increase of more than $20 \%$ of the $r C B F$ is present only in the primary sensorimotor cortex contralateral to the voluntarily moved fingers, but mirror movements were found during the motor task. The areas of increased $r C B F$ are almost identical to the MEP topographical maps for the thenar muscles of both patients.

mal threshold. Stimulus sites were selected using a grid with positions 1 or $2 \mathrm{~cm}$ apart in reference to $\mathrm{Cz}$ (the international 10-20 system).

During voluntary tonic contraction in the right hand at $10 \%$ maximum force by patient 1 and in the left at the maximum by patient 2 who could not appropriately adjust the force because of his paresis, we studied the silent periods in both their thenar muscles (the voluntary contraction and mirror movement sides) using transcranial magnetic stimulation given over the contralateral or ipsilateral hand motor area of the voluntary contraction side. Two to three stimuli were given at $70 \%$ to $100 \%$ of the maximum stimulator output in $10 \%$ increments. The EMGs for each intensity were rectified and averaged. The duration of the silent period was estimated from the time during which the EMG level was below the mean amplitude of the EMG before transcranial magnetic stimulation.

We also investigated the ipsilateral silent period in unilaterally contracted thenar muscle using transcranial magnetic stimulation over the ipsilateral hand motor area in six right handed healthy volunteers (three men, three women; ages 21-36) who gave their informed consent for the study.

\section{$\mathrm{H}_{2}{ }^{15} \mathrm{O}$-PET ACTIVATION STUDY}

Regional cerebral blood flow ( $\mathrm{rCBF}$ ) was measured repeatedly in both patients by the PET autoradiographic method. First a bolus injection of $1.5 \mathrm{GBq} \mathrm{H}_{2}{ }^{15} \mathrm{O}$ was given, followed immediately by two minutes of scanning with a PET camera, the HEADTOME-IV (Shimadzu, Kyoto), for two tasks: (1) a voluntary motor task, in the right hand for patient 1 and in the left for patient 2 , in which the thumb was repeatedly touched against the tips of all the other fingers at a rate of about two touches per second, and (2) the resting state. For both tasks, the patients were asked to look at a white spot on the video screen in front of them. Details of the data acquisition and analysis methods have been described elsewhere. ${ }^{7}$ Normalised and subtracted rCBF images were registered to the MRI by the $3 D$ shift and rotation for anatomical reference, ${ }^{8}$ and change in the rCBF was evaluated.

\section{Results}

In both patients, transcranial magnetic stimulation on one side elicited MEPs in the contralateral and ipsilateral thenar muscles with similar latencies $(21.5$ and $20.7 \mathrm{~ms}$ in patient 1 and 21.1 and $21.4 \mathrm{~ms}$ in patient 2 ) and with 

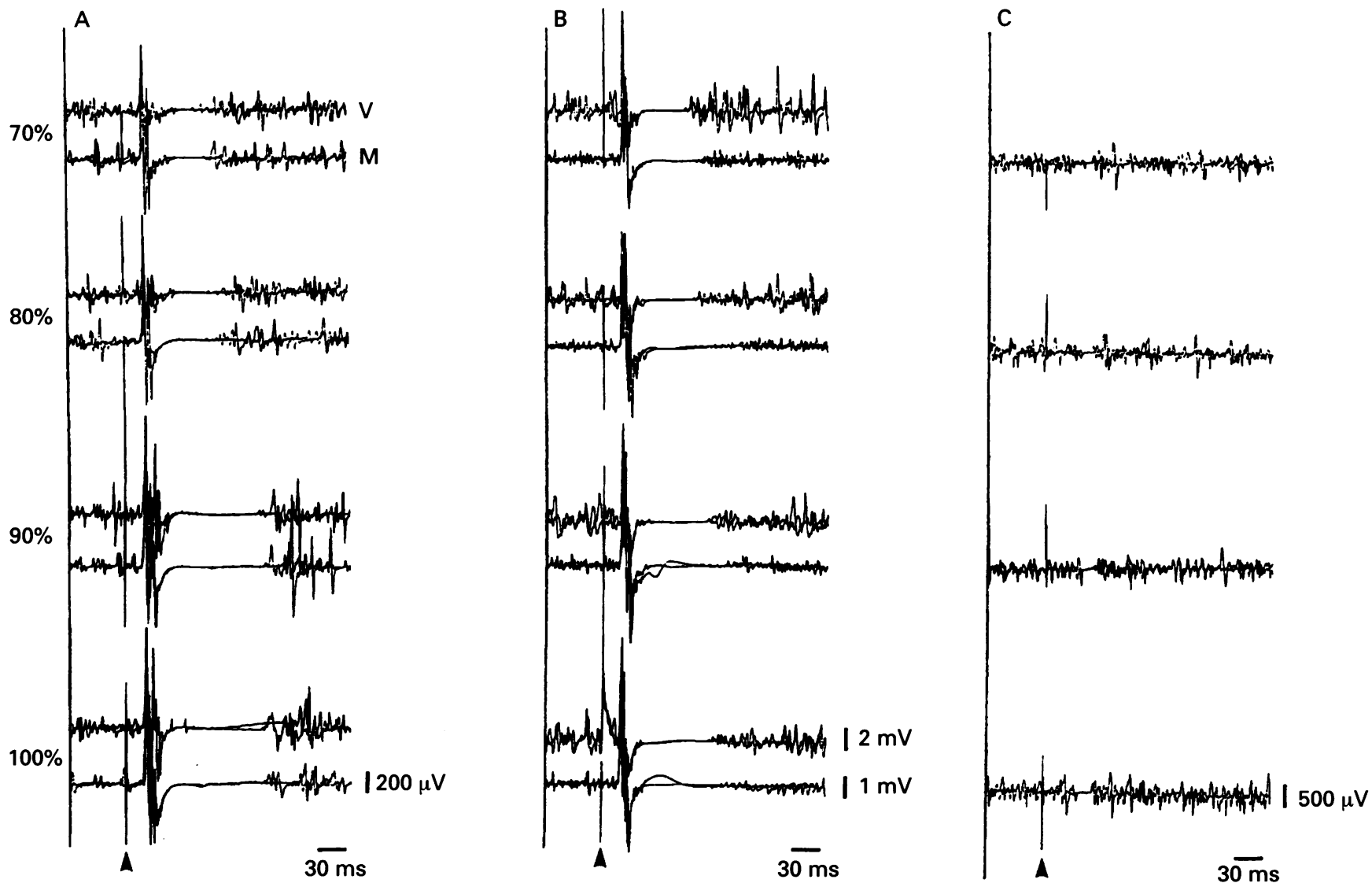

Figure 2 Silent periods induced by transcranial magnetic stimulation over the hemisphere contralateral to the unilateral voluntary contraction side in patient 1 (A) and 2 (B), and ipsilateral silent periods in a normal subject (C). Arrowheads indicate transcranial magnetic stimulation. Trwo recordings were superimposed. In each pair of recordings, the upper and lower traces respectively show recordings on the voluntary contraction ( $V)$ and mirror movement (M) sides. Note that not only the contralateral and ipsilateral MEP latencies, but the durations of the silent periods are almost equal.

Figure 3 Transcranial magnetic stimulation given over the hemisphere ipsilateral to the voluntary contraction side with maximum stimulator output (A: patient $1 ; B$. patient 2). It did not induce silent periods bilaterally, but did elicit MEPs on both sides. similar minimal thresholds $(62 \%$ and $60 \%$ in patient 1 and $62 \%$ and $62 \%$ in patient 2 ). Topographical cortical mapping showed that the area over which transcranial magnetic stimulation elicited MEPs in the ipsilateral thenar muscle was almost identical to that in the contralateral muscle (fig 1).

Transcranial magnetic stimulation over the hand motor area contralateral to the voluntary contraction side always induced silent period after MEP on both the voluntary contraction and mirror movement sides. Both silent periods showed complete cessation of EMG activities. These lasted almost the same time, and became longer with the increase in transcranial magnetic stimulation intensity (fig $2 \mathrm{~A} \mathrm{~B}$ ). The durations of the silent period induced by the maximum intensity transcranial magnetic stimulation were $106 \mathrm{~ms}$ and $114.8 \mathrm{~ms}$ on the contralateral voluntary and ipsilateral mirror movement sides for patient 1 , and $84.6 \mathrm{~ms}$ and $89.4 \mathrm{~ms}$ for patient 2 .

The ipsilateral silent periods in the normal subjects often showed incomplete cessation or partial reduction of EMG activities, the mean duration being 24.0 (SD 4.70) ms (range $16 \cdot 2-30.4 \mathrm{~ms}$ ) at maximum stimulator output (fig 2C).

In both patients, transcranial magnetic stimulation given over the hand motor area contralateral to the mirror movement side often failed to induce silent periods on either side, whereas MEPs always were elicited on both sides (fig 3 ).

An $\mathrm{H}_{2}{ }^{15} \mathrm{O}$-PET activation study showed that the intended unilateral finger movement produced an increase of more than $20 \%$ of the rCBF only in the contralateral primary sensori-

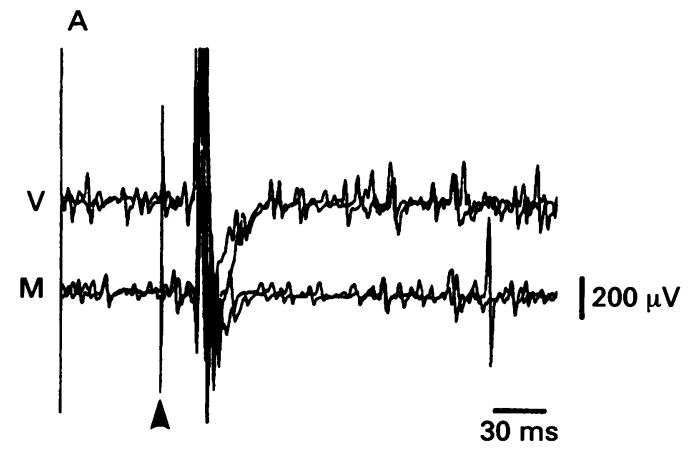

B

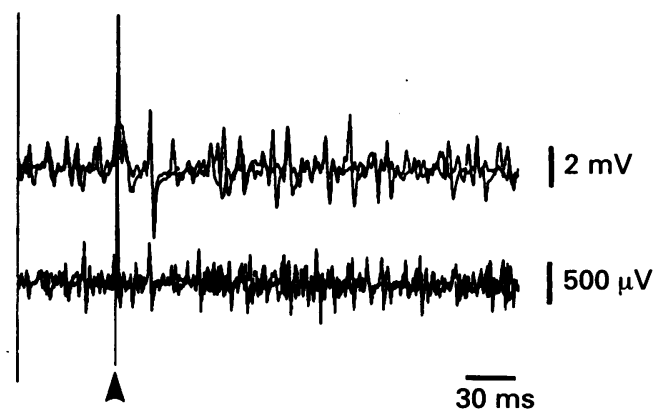


motor cortex, but mirror movements occurred in both patients during the motor task (fig 1).

\section{Discussion}

In both patients, transcranial magnetic stimulation on one side elicited MEPs in both thenar muscles with similar latencies, minimal thresholds, and cortical topographies. These findings suggest that there are connections between the primary motor cortex and ipsilateral thenar muscle, which can conduct efferent volleys as fast as the crossed corticospinal tract in normal subjects. Similar results were reported in studies of patients with mirror movements; ${ }^{1356}$ but, in all except a patient with Klippel-Feil syndrome, ${ }^{3}$ it is not clear whether the mirror movements are actually induced through the ipsilateral pathway. To determine whether the same side of the cortex as that which generates voluntary movements on the contralateral side produces mirror movements as transcranial magnetic stimulation elicited ipsilateral MEPs, we investigated the silent period in the ongoing EMG activities of mirror movements.

The silent period is the inhibition of ongoing voluntary EMG activities after the MEP elicited by transcranial magnetic stimulation. Although the segmental inhibitory mechanism may play a part in the origin of the first $50 \mathrm{~ms}$ of the normal contralateral silent period, the later part may be generated by the cortical inhibitory mechanism..$^{10}$ Schnitzler and Benecke showed that in two patients with focal isolated ischaemic lesions of the primary motor cortex, transcranial magnetic stimulation elicited MEPs, but failed to induce silent periods in the clinically affected contralateral muscles. ${ }^{11}$ They concluded that both the early and late phases of the silent period are generated in the primary motor cortex.

In our patients, transcranial magnetic stimulation over the hand motor area contralateral to the voluntarily contracted muscle always induced equally long silent periods on both the voluntary contraction and mirror movement sides. Silent periods on the ipsilateral mirror movement side were much longer than the normal ipsilateral silent period which is speculated to be due to transcallosal inhibition of the contralateral motor cortex. ${ }^{12}{ }^{13}$ The very similar silent periods produced on the voluntary contraction and mirror movement sides suggest that mirror muscle activities were inhibited by the same mechanism that inhibits contralateral voluntary muscle activities. Because of the more than $50 \mathrm{~ms}$ duration, those inhibitions are considered to occur at the cortical level. We therefore suggest that mirror activities were inhibited in the cortex on the mirror movement side. This is supported by the fact that transcranial magnetic stimulation given contralateral to the mirror movement side did not inhibit mirror muscle activities. Schnitzler and Benecke suggested that selec- tive damage of inhibitory interneurons in the primary motor cortex can cause a complete loss of the silent period in the contralateral muscle without affecting the MEP. ${ }^{11}$ In our patients, however, we attribute a loss of the silent periods, when transcranial magnetic stimulation was given over the contralateral primary motor cortex of the mirror movement side, not to the impaired inhibitory mechanism for silent period in the stimulated cortex, but to an absence of the neural activities generating mirror movements in the cortex. Possibly, the muscle activities which were not inhibited originated in the motor cortex contralateral to transcranial magnetic stimulation. This explanation is well supported by the results of our PET study: more than $20 \%$ of the $\mathrm{rCBF}$ increase was only in the sensorimotor cortex contralateral to the voluntarily moved fingers, but mirror movements were seen during the motor task. The side of the rCBF increase was identical to the cortical side on which transcranial magnetic stimulation induced silent periods bilaterally and vice versa.

We therefore consider that the same side of the motor cortex that generates voluntary movements has a major role in the generation of mirror movements which may be induced through the ipsilateral uncrossed corticospinal pathway as suggested by the ipsilateral MEP results.

We are grateful to Dr Chiaki Inoue, Dr Masahiko Yamamoto, and Dr Michio Senda for their help.

1 Danek A, Heye B, Schoedter R. Cortically evoked motor responses in patients with Xp22.3-linked Kallmann's syndrome and in female gene carriers. Ann Neurol 1992; 31:299-304.

2 Shibasaki $\mathrm{H}$, Nagae $\mathrm{K}$. Mirror movement: application of movement-related cortical potentials. Ann Neurol 1984; 15:299-302.

3 Farmer SF, Ingram DA, Stephens JA. Mirror movements studied in a patient with Klippel-Feil syndrome. $\mathscr{F}$ Physiol 1990;428:467-84.

4 Nass R. Mirror movement asymmetries in congenital hemiparesis: the inhibition hypothesis revisited. Neurology 1985;35:1059-62.

5 Britton TC, Meyer B-U, Benecke R. Central motor pathways in patients with mirror movements. $f$ Neurol Neurosurg Psychiatry 1991;54:505-10.

6 Cohen LG, Meer J, Tarkka I, et al. Congenital mirror movements. Abnormal organization of motor pathways in two patients. Brain 1991;114:381-403.

7 Senda M, Kanno I, Yonekura Y, et al. Comparison of anatomical standardization methods regarding the sensorimotor foci localization and between-subject variation in rimotor foci localization and between-subject variation in $\mathrm{H}_{2}{ }^{15}$ O PET activation, a three
Ann Nucl Med 1994;8:201-7.

8 Senda M. Mapping cortical functions using PET activation technique. In: Sugishita M, ed. New horizons in neuropsytechnique. In: Sugishita M, ed. New horizons

9 Fuhr P, Agostino R, Hallett M. Spinal motor neuron excitability during the silent period after cortical stimulation. Electroencephalogr Clin Neurophysiol 1991;81: 257-62.

10 Inghilleri M, Berardelli A, Cruccu G, Manfredi M. Silent period evoked by transcranial stimulation of the human cortex and cervicomedullary junction. If Physiol 1993; 466:521-34

11 Schnitzler A, Benecke $R$. The silent period after transcranial magnetic stimulation is of exclusive cortical origin: evidence from isolated cortical ischemic lesions in man. Neurosci Lett 1994;180:41-5.

12 Wassermann EM, Fuhr P, Cohen LG, Hallett M. Effects of transcranial magnetic stimulation on ipsilateral muscles. Neurology 1991;41:1795-9.

13 Meyer B-U, Röricht S, Gräfin von Einsiedel H, Kruggel F, Weindl A. Inhibitory and excitatory interhemispheric transfers between motor cortical areas in normal humans and patients with abnorm 